\title{
Parameterisation and application of dynamic energy budget model to sea cucumber Apostichopus japonicus
}

\author{
Jeffrey S. Ren ${ }^{1}$, Jeanie Stenton-Dozey ${ }^{1}$, Jihong Zhang ${ }^{2,3, *}$ \\ ${ }^{1}$ National Institute of Water and Atmospheric Research, 10 Kyle Street, PO Box 8602, Christchurch 8440, New Zealand \\ ${ }^{2}$ Yellow Sea Fisheries Research Institute, Chinese Academy of Fishery Sciences, 106 Nanjing Road, Qingdao 266071, PR China \\ ${ }^{3}$ Function Laboratory for Marine Fisheries Science and Food Production Processes, \\ Qingdao National Laboratory for Marine Science and Technology, 1 Wenhai Road, Aoshanwei, Jimo, Qingdao 266200, PR China
}

\begin{abstract}
The sea cucumber Apostichopus japonicus is an important aquaculture species in China. As global interest in sustainable aquaculture grows, the species has increasingly been used for co-culture in integrated multitrophic aquaculture (IMTA). To provide a basis for optimising stocking density in IMTA systems, we parameterised and validated a standard dynamic energy budget (DEB) model for the sea cucumber. The covariation method was used to estimate parameters of the model with the DEBtool package. The method is based on minimisation of the weighted sum of squared deviation for datasets and model predictions in one single-step procedure. Implementation of the package requires meaningful initial values of parameters, which were estimated using non-linear regression. Parameterisation of the model suggested that the accuracy of the lower $\left(T_{\mathrm{L}}\right)$ and upper $\left(T_{\mathrm{H}}\right)$ boundaries of tolerance temperatures are particularly important, as these would trigger the unique behaviour of the sea cucumber for hibernation and aestivation. After parameterisation, the model was validated with datasets from a shellfish aquaculture environment in which sea cucumbers were co-cultured with the scallop Chlamys farreri and Pacific oyster Crassostrea gigas at various combinations of density. The model was also applied to a land-based pond culture environment where the sea cucumber underwent a fast growth period in spring and non-growth periods during winter hibernation and summer aestivation. Application of the model to datasets showed that the model is capable of simulating the physiological behaviour of the sea cucumber and responds adequately to the wide range of environmental and culture conditions.
\end{abstract}

KEY WORDS: Sea cucumber - Dynamic energy budget model - DEB model - Parameterisation · Covariation method $\cdot$ Application

\section{INTRODUCTION}

The sea cucumber Apostichopus japonicus, a commercially valuable species, is commonly found in shallow intertidal habitats off the west Pacific Ocean. The vertical distribution ranges from intertidal areas down to depths of 20-30 m (Chen 1990). Culture of the species started in the early 1980s after success of the technique in commercial hatcheries and seed

\footnotetext{
${ }^{*}$ Corresponding author: zhangjh@ysfri.ac.cn
}

production in China (Liao 1997). Since then, sea cucumber farming has become an important part of mariculture and the scale of the industry has increased dramatically (Chen 2003, Zhang et al. 2015).

Sea cucumbers are surface-deposit feeders. They are not only commercially important, but also play an important role in coastal ecosystems. They ingest and assimilate a large amount of organic matter deposited on the seafloor prior to extensive microbial pro-

(C) The authors 2017. Open Access under Creative Commons by Attribution Licence. Use, distribution and reproduction are unrestricted. Authors and original publication must be credited. 
cessing. Sea cucumbers have often been called the earthworms of the sea, because they are responsible for the extensive shifting and mixing of the substrate and recycling of detrital matter (Lauerman et al. 1997, Miller et al. 2000, Yang et al. 2005). They grind sedimentary organic matter into finer particles, turning over the top layers of sediment in lagoons, reefs and other habitats, and allowing the penetration of oxygen (Lauerman et al. 1997). Sea cucumbers are also important in determining habitat structure for other species and can represent a substantial portion of the ecosystem biomass (Sibuet \& Segonzac 1985).

The ability to turn biodeposition into valuable flesh product and being ecologically complementary with many aquaculture species make the sea cucumber an important species for co-cultures in integrated multitrophic aquaculture (IMTA) (e.g. Zhou et al. 2006, Slater et al. 2009, Zamora et al. 2014, Yuan et al. 2015). Over the past decade, experimental and commercial trials have been conducted to co-culture it with other species to improve water quality and mitigate environmental impacts (e.g. Li et al. 2001, Yang et al. 2001, Yu et al. 2014). These studies have shown an increase in system productivity and improvement of environmental conditions. Despite promising results, these experiments were based on the traditional technique of trial and error and experimentation, and the right proportion of stocking density for each co-cultured species could not be satisfactorily estimated. Maximum ecological and economic benefits of IMTA systems can only be achieved with the optimal density at which species are physiologically and ecologically complementary (Chopin et al. 2008, Barrington et al. 2009). The growth rate of the sea cucumber depends on the rates of biodeposition from other species within an IMTA system where the stocking density of cocultured species can be optimised by means of mathematical models. Recently, a model was developed to optimise production in IMTA systems (Ren et al. 2012). A dynamic energy budget (DEB) model of the sea cucumber is one of the sub-models of the larger IMTA model.

Although DEB modelling has gained increasing popularity, most modelling development has been focused on shellfish to improve shellfish aquaculture (e.g. Ren \& Schiel 2008, Rosland et al. 2009, AlunnoBruscia et al. 2011), while little attention has been paid to deposit-feeders. The objective of this study is to develop a DEB model of the sea cucumber for subsequent application in IMTA systems. We followed a standard DEB model and the main focus of the study was on its parameterisation and validation.

\section{MATERIALS AND METHODS}

\section{Model structure and parameterisation}

A DEB model is based on a generic theory that biological organisms share common physiological behaviours (Kooijman 2010). The same functional structure could therefore be applied to different species with species-specific parameter values. Although some effort has been made to extend the model by coping with varying food quantity and quality (Saraiva et al. 2012), the standard form of the DEB model has been widely adopted to study energetics and growth of aquaculture species, particularly bivalves (e.g. Ren \& Schiel 2008, Bourlès et al. 2009, Rosland et al. 2009). Our primary purpose was to develop and validate a simple model of the sea cucumber, which can be incorporated into a larger IMTA model for optimising production of IMTA. Therefore, a standard DEB model was ideal for this purpose. The model structure, including functional responses and energy allocation, has been described in detail (van der Meer 2006, Ren \& Schiel 2008), and we only focus on parameterisation here. The same symbols were used as those in the standard DEB model.

Estimation of model parameters depends on the quantity and quality of the experimental data. However, comprehensive data are not usually available for most species. For some species with sufficient datasets, the estimated values of parameters are often inconsistent between datasets due to variation in experimental conditions (Saraiva et al. 2011). To account for this problem, van der Veer et al. (2006) suggested a standard procedure using all available datasets by means of weighted non-linear regression. Recently, a covariation method has been developed to estimate parameters of a standard DEB model. It is based on the simultaneous minimisation of the weighted sum of squared deviations between datasets and predictions in one single-step procedure (Lika et al. 2011). For the present modelling exercise, we adopt this method to estimate parameters of the sea cucumber model using the DEBtool package (www.bio.vu.nl/thb/deb/deblab/). Implementation of the method requires 2 types of datasets including real data and pseudo-data. The real data consist of zero-variate and univariate data, which are from actual observations of the sea cucumber at known temperatures and food conditions. The univariate data of length versus age are shown in Fig. 1. The data reflects the mean growth of the species from post-settlement at the natural environment. Lack of length at early stages in univariate data may 


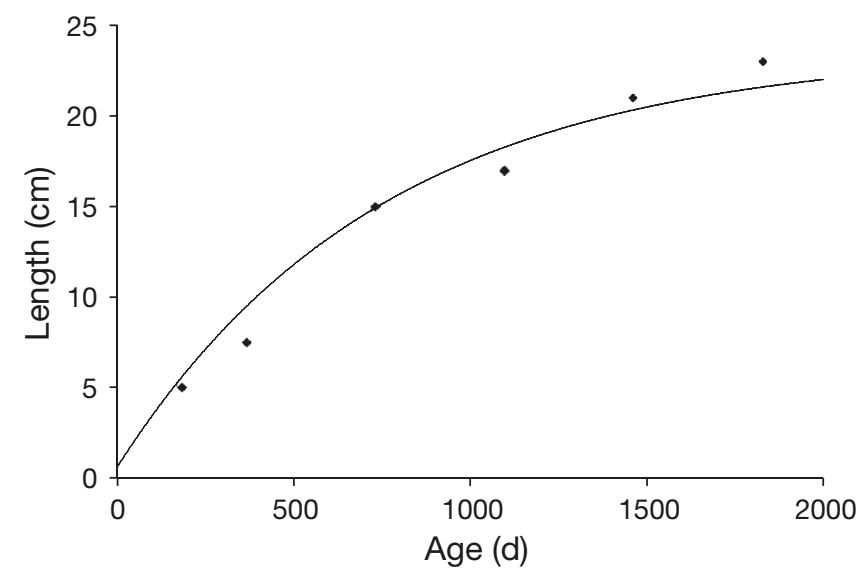

Fig. 1. Simulation of sea cucumber (Apostichopus japonicus) length using the primary parameters of the dynamic energy budget (DEB) model (line). Dots are observations and the line is the fitted growth curve

not considerably compromise the estimation of final parameter values, because zero-variate data consists of information on length at birth. The pseudo-data are a set of values of primary or compound parameters usually for a generalised animal obtained from a large collection of estimated parameters for a wide variety of species (Kooijman 2010, Saraiva et al. 2011). The concept of pseudo-data is used to avoid an unrealistic combination of parameters and to maintain the rules for covariation of the parameter values implied by physical laws (Saraiva et al. 2011). The pseudo-data include parameters for the Arrhenius temperature $\left(T_{\mathrm{A}}\right)$, volume-specific somatic maintenance coefficient $\left[p_{\mathrm{M}}\right]$ and shape coefficient $\left(\delta_{\mathrm{M}}\right)$. Because the initial values determine whether the results are meaningful, these values should be as close to 'real' as possible. For the present study, we estimated the parameter values from experimental data for Apostichopus japonicus, rather than generalised animal parameter values. A procedure proposed by van der Meer (2006) was followed to estimate initial values of these parameters. DEB theory assumes that all rates (e.g. assimilation, catabolic and maintenance rates) can be described by an Arrhenius relationship using a single Arrhenius temperature within a speciesspecific tolerance range. The estimation of parameters in the Arrhenius equation $\left(T_{\mathrm{A}}, T_{\mathrm{AL}}, T_{\mathrm{AH}}, T_{\mathrm{L}}\right.$ and $T_{\mathrm{H} i}$ see Table 2 for definitions) was based on data from physiological experiments within a range of temperatures ( $\mathrm{Li}$ et al. 2002). The volume-specific somatic maintenance coefficient is the ratio of the costs of maintenance to structural volume synthesis. It was indirectly estimated from changes in energy content during aestivation over time (Zhou et al. 2006).
The shape coefficient is used for conversion between length and biovolume. It was estimated from the relationship between length and wet mass by assuming that the density of the biovolume equals $1 \mathrm{~g} \mathrm{~cm}^{-3}$. The DEB assumes that the energy allocation follows the $\kappa$-rule, which assumes that a fixed proportion $\kappa$ of the energy from the reserves goes to maintenance and growth. The remaining fraction $1-\kappa$ goes to development and reproduction. The initial value for the fraction of energy utilisation rate spent on maintenance plus growth $(\kappa)$ can be determined from reproduction information, i.e. the maximum gonadal mass fraction relative to other tissue (van der Veer et al. 2006). Based on reproduction information (Chen 1990), the value was estimated following van der Veer et al. (2006). The use of an area-specific assimilation rate, $\left\{\dot{p}_{\mathrm{Am}}\right\}$, is no longer needed with the covariation method, because it is derived from some additional parameters. For comparison, we still estimate its value with this method. The real data and pseudo-data are listed in Table 1.

There is still a lack of data for the initial values of a few parameters including maturity at birth $\left(E_{\mathrm{Hb}}\right)$, maturity at metamorphosis $\left(E_{\mathrm{Hj}}\right)$, maturity at puberty $\left(E_{\mathrm{Hp}}\right)$ and Weibull aging acceleration $\left(h_{\mathrm{a}}\right)$. Following the methodology of Lika et al. (2011), default values were initially used and subsequently tuned until all parameters could be determined by observational data. It should be noted that the Gompertz stress coefficient $\left(s_{\mathrm{G}}\right)$ could be particularly important for the sea cucumber, because this species would undergo winter hibernation and summer aestivation, and may also eviscerate when an external stress occurs. This would cause drastic consequences on its energetics. The Gompertz stress coefficient controls the mean lifespan. Increasing the value decreases the mean lifespan, but increases survival at young age. According to Kooijman (2010), negative values can occur if damage-inducing compounds can be degraded. The sea cucumber would fall in this category and hence a negative value is assigned for $s_{\mathrm{G}}$. The final values of the parameters are listed in Table 2.

\section{Validation datasets}

The model was validated using a few datasets collected from growth experiments of the sea cucumber. The first dataset was from a co-culture experiment to determine the growth performance of the sea cucumber with shellfish (Zhou et al. 2006). In this experiment, sea cucumbers were co-cultured with either scallops Chlamys farreri or oysters Crassostrea 
Table 1. Data used in parameter estimation procedure. gWW: grams of wet weight

\begin{tabular}{|c|c|c|c|c|}
\hline Symbol & Value & Unit & Definition & Source \\
\hline \multicolumn{5}{|c|}{ Zero-variate } \\
\hline$a_{\mathrm{b}}$ & 8 & $\mathrm{~d}$ & Age at birth $\left(20^{\circ} \mathrm{C}\right)$ & Qiu et al. (2015) \\
\hline$a_{\mathrm{p}}$ & 300 & d & Age at puberty $\left(20^{\circ} \mathrm{C}\right)$ & Yamana et al. (2010) \\
\hline$a_{\mathrm{m}}$ & $7 \times 365$ & $\mathrm{~d}$ & Lifespan & Sun $(2013)$ \\
\hline$L_{\mathrm{b}}$ & 0.08 & $\mathrm{~cm}$ & Length at birth & Ito \& Kitamura (1998), Qiu et al. (2015) \\
\hline$L_{\mathrm{p}}$ & 7.5 & $\mathrm{~cm}$ & Length at puberty & Hamano et al. (1989), Ito \& Kitamura (1998) \\
\hline$L_{\mathrm{i}}$ & 29.7 & $\mathrm{~cm}$ & Maximum length & Yamana et al. (2010), Hamano et al. (1989) \\
\hline$W_{\mathrm{b}}$ & $0.064 L_{\mathrm{b}}^{3}$ & gWW & Wet weight at birth & Sun (2013) \\
\hline$W_{\mathrm{p}}$ & $0.064 L_{\mathrm{p}_{3}}^{3}$ & gWW & Wet weight at puberty & Sun (2013) \\
\hline$W_{\mathrm{i}}$ & $0.064 L_{\mathrm{i}}^{3}$ & gWW & Ultimate weight & Sun (2013) \\
\hline \multicolumn{5}{|c|}{ Pseudo-data } \\
\hline$\kappa$ & 0.85 & - & Allocation fraction & Chen (1990) \\
\hline$\kappa_{\mathrm{G}}$ & 0.95 & - & Growth efficiency & Liu et al. (1996) \\
\hline$\dot{v}$ & 0.02 & $\mathrm{~cm}$ & Energy conductance & Kooijman (2010) \\
\hline$\left[\dot{p}_{\mathrm{M}}\right]$ & 4.8 & $\mathrm{~J} \mathrm{~cm}^{-3} \mathrm{~d}^{-1}$ & Volume-specific somatic maintenance & Liu et al. (1996) \\
\hline$\delta_{\mathrm{m}}$ & 0.28 & - & Shape coefficient & Sun $(2013)$ \\
\hline$T_{\mathrm{A}}$ & 7300 & $\mathrm{~K}$ & Arrhenius temperature & Li et al. (2002) \\
\hline$\left\{\dot{p}_{\mathrm{Am}}\right\}$ & 154 & $\mathrm{~J} \mathrm{~cm}^{-3} \mathrm{~d}^{-1}$ & Maximum area-specific assimilation rate & Zhou et al. (2006) \\
\hline \multicolumn{5}{|c|}{ Univariate } \\
\hline Growth & ata & yr vs. cm & Time vs. length & Sun (2013), Qiu et al. (2015) \\
\hline
\end{tabular}

gigas in the shellfish farm of Sishili Bay $\left(37^{\circ} 32^{\prime} \mathrm{N}\right.$, $120^{\circ} 30^{\prime} \mathrm{E}$ ) off Shandong Peninsula, China. A few different combinations of co-culture practices were used.

For the present study, we chose 2 datasets from cocultures of sea cucumbers with scallops and oysters, respectively. For co-culture of sea cucumber with scallops, 10 sea cucumbers and 350 scallops were placed in each lantern net. The initial average body weight of the sea cucumber was $56.9 \mathrm{~g}$ and the scal- lop shell length was $2.3 \mathrm{~cm}$. For co-culture of sea cucumbers with oysters, the animals were cultured at a density of 10 sea cucumbers with 120 oysters. The initial average body weight of sea cucumbers was $39 \mathrm{~g}$ and the oyster length $7.7 \mathrm{~cm}$. The experiment lasted $\sim 8$ mo. The body weights of the sea cucumbers were measured monthly for the first $3 \mathrm{mo}$ and at the end of the experiment (see details in Zhou et al. 2006). Temperature was not recorded during the experiment. Because the variation of water tempera-

Table 2. Final dynamic energy budget (DEB) model parameters for sea cucumber Apostichopus japonicus and other parameters

\begin{tabular}{|lccll|}
\hline Symbol & Value & Unit & Definition & Source \\
\hline$\kappa$ & 0.85 & - & Catabolic flux to growth and maintenance & Chen (1990) \\
$\kappa_{\mathrm{R}}$ & 0.8 & - & Reproduction efficiency & This study \\
$\left\{\dot{p}_{\mathrm{Am}}\right\}$ & 129.5 & $\mathrm{~J} \mathrm{~cm}^{-3} \mathrm{~d}^{-1}$ & Area-specific maximum assimilation rate & This study \\
{$\left[\dot{p}_{\mathrm{M}}\right]$} & 12.1 & $\mathrm{~J} \mathrm{~cm}^{-3} \mathrm{~d}^{-1}$ & Volume-specific maintenance rate & This study \\
{$\left[E_{\mathrm{m}}\right]$} & 4300 & $\mathrm{~J} \mathrm{~cm}^{-3}$ & Maximum storage density & This study \\
{$\left[E_{\mathrm{G}}\right]$} & 1700 & $\mathrm{~J} \mathrm{~cm}^{-3}$ & Volume-specific costs for structure & This study \\
$T_{\mathrm{A}}$ & 5500 & $\mathrm{~K}$ & Arrhenius temperature & Li et al. (2002) \\
$T_{\mathrm{AL}}$ & 70000 & $\mathrm{~K}$ & Rate of decrease at lower boundary & Yang et al. (2005) \\
$T_{\mathrm{AH}}$ & 35500 & $\mathrm{~K}$ & Rate of decrease at upper boundary & Yang et al. (2005) \\
$T_{\mathrm{L}}$ & 273 & $\mathrm{~K}$ & Lower boundary of tolerance range & Zhang et al. (2004), Yang et al. (2005) \\
$T_{\mathrm{H}}$ & 290 & $\mathrm{~K}$ & Upper boundary of tolerance range & Liu et al. (1996), Yang et al. (2005) \\
$\delta_{\mathrm{m}}$ & 0.28 & - & Shape coefficient & Sun (2013) \\
$\dot{v}$ & 0.03 & $\mathrm{~cm} \mathrm{~d}$ & Energy conductance & This study \\
$E_{\mathrm{Hb}}$ & 4.7 & $\mathrm{~J}$ & Maturity at birth & This study \\
$E_{\mathrm{Hp}}$ & 7200 & $\mathrm{~J}$ & Maturity at puberty & This study \\
$E_{\mathrm{Hj}}$ & 5.1 & $\mathrm{~J}$ & Maturity at metamorphosis & This study \\
$h_{\mathrm{a}}$ & $1.4 \times 10^{-7}$ & $\mathrm{~J}$ & Weibull aging acceleration & This study \\
$S_{\mathrm{G}}$ & -0.2 & - & Gompertz stress coefficient & This study \\
\hline
\end{tabular}


ture in the bay follows an annual pattern, the annual cycle data were used for the model simulation.

The second dataset was obtained from a landbased growth experiment near Qingdao off Shandong Peninsula, China (Yu \& Song 1999). The experiment lasted 1 yr. Juveniles with an average body weight of $\sim 2.5 \mathrm{~g}$ were transplanted from a hatchery to a culture pond. The animals were fed with artificial food made of algae meal, but feeding was suspended during the aestivation period in summer. Body weight and water temperature were measured monthly.

\section{SIMULATIONS AND RESULTS}

Temperature and food density are forcing variables used to run the model. There was significant difference in water temperature between the experiments (ANOVA, p < 0.01). It was considerably higher in the land-based experiment than in the sea-based ones from February through August. This pattern reversed during the rest of the year (Fig. 2).

The model was applied to the first dataset. The optimal fit of the model to the observation resulted in $f$-values of 0.51 and 0.62 , respectively under coculture practices with oysters and scallops (Fig. 3). (Because the functional response $(f)$ describes the relationship between food uptake and density, $f$-value is an indicator of food availability). This suggested that food in the co-culture with scallops was more abundant than with oysters. The daily growth rates were 1.3 and $1.6 \%$ for co-culture with oysters and scallops, respectively. The model simulations appropriately reflected differences in environmental conditions, particularly food availability.

The model was also applied to the growth of the sea cucumber in the land-based culture ( $2^{\text {nd }}$ dataset). The model with the fixed $f$-value of 0.59 would result in prediction being consistent with the observation (Fig. 4), which indicates that the model can reasonably reproduce the physiological behaviour of the sea cucumber. Little growth occurred during winter and summer months, but the growth was high during the spring months when the temperature was optimal for growth. When temperature was low in winter, animals considerably reduced feeding and hibernation may have occurred. Similarly, when water temperature rises from mid-June, aestivation was induced and animals stopped feeding until September when temperature decreases to the growth range. Both situations resulted in ceasing growth of structural volume and the energy requirement for mainte-

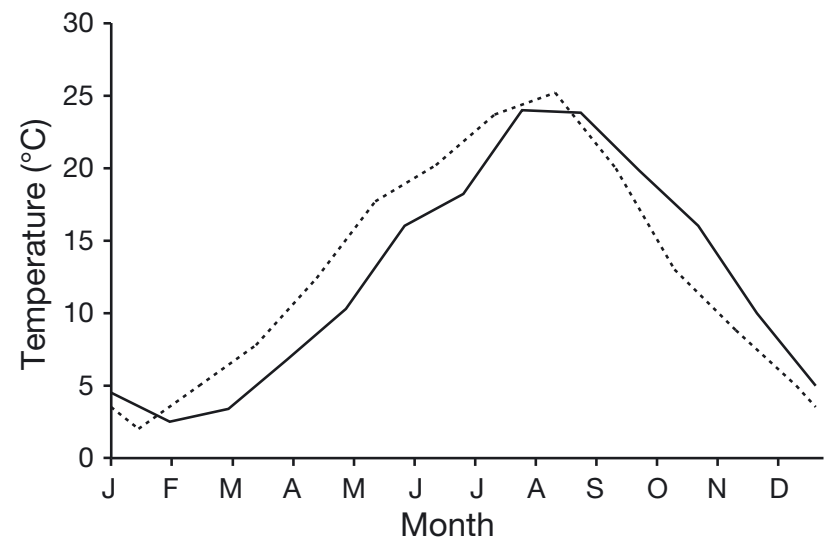

Fig. 2. Annual variation of temperatures in Sishili Bay (solid line) and culture pond (dotted line) off Shandong Peninsula, China

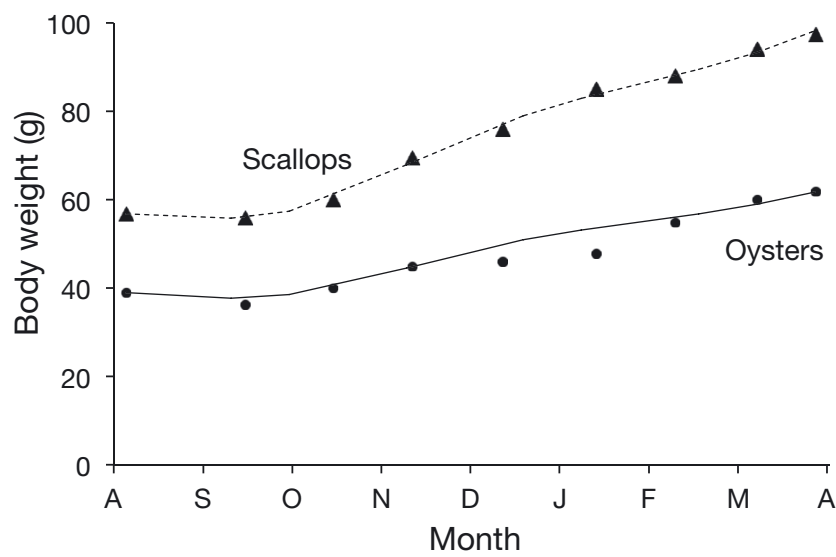

Fig. 3. Comparison between the observed (dots and triangles) and simulated (solid and dashed lines) growth of sea cucumber Apostichopus japonicus in the co-culture environment during August 2000-April 2001. Solid and dashed lines are the modelled body weight from co-cultures with oysters Crassostrea gigas and scallops Chlamys farreri, respectively

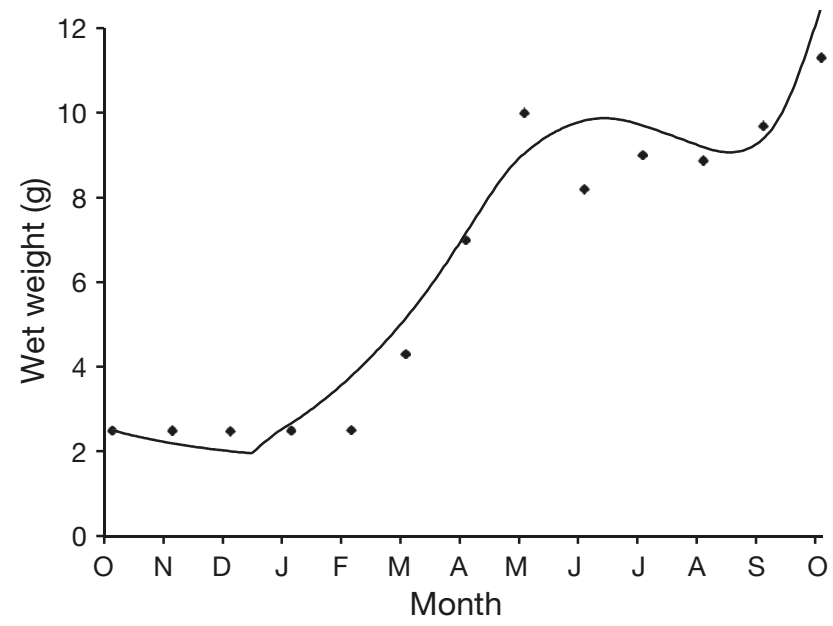

Fig. 4. Comparison between observed (dots) and simulated (line) growth of sea cucumber Apostichopus japonicus from a land-based culture system during October 1981-October 1982 
nance is met from reserves, which caused the decline of body weight due to this net negative energy flow. Overall, food availability is similar to that in the scallop co-culture environment, but slightly higher than in the oyster co-culture system.

\section{DISCUSSION}

The present modelling exercise aimed to parameterise and validate a standard DEB model of the sea cucumber for subsequent application in IMTA ecosystems. The model parameters were mainly estimated using the covariation method. Application of the model to data proved successful, as the model is capable of reproducing the observed patterns of growth in different environmental and culture conditions, including co-culture with oysters and scallops, and land-based pond culture.

In parameterisation of the DEB model, there are a few noticeable differences of some parameter values estimated between the covariation method and weighted non-linear regression. The maximum areaspecific assimilation, $\left\{\dot{p}_{\mathrm{Am}}\right\}$, is lower, and volume-specific somatic maintenance, $\left[\dot{p}_{M}\right]$, is higher than the initial values. This suggests that the ability of the sea cucumber to capture food is low but the animal spends more energy for somatic maintenance than those from experimental data. Similarly, the reserve capacity $\left[E_{\mathrm{m}}\right]$ from the covariation method is higher than the initial value. These differences may largely be due to variations in experimental conditions, which magnify the estimation of parameter values. In addition, parameter values with a poorer fit may have much more physical realism, because observations cannot include all possible aspects of energy budgets (Lika et al. 2011). For example, the maximum reserve $\left[E_{\mathrm{m}}\right]$ cannot directly be measured and is usually estimated from starvation experiments, but such measurement depends on the initial condition of an organism and experimental conditions over the starvation period. In addition, the sea cucumber is extremely sensitive to changes in environmental conditions, particularly temperature, which can induce atypical behaviours including evisceration, hibernation and aestivation. Therefore, given sufficient information on the required data, the final parameter values estimated from the covariation method would appropriately reflect the behaviour of the species.

The use of the covariation method in parameter estimation is a considerable improvement over previous ones. It is based on minimisation of the weighted sum of squared deviation for datasets and model pre- dictions in one single-step procedure (Lika et al. 2011). It also includes physiological constraints by introducing pseudo-data to reduce deviation of parameters related to variations in experimental datasets. Development of the covariation method would help increase the application of the DEB model. A standard DEB model provides a framework for functional structure which is applicable to different species with species-specific parameter values, while the covariation method offers a general technique for parameterisation of the DEB model. However, implementation of the method requires completeness of zerovariate data including age, length and weight at birth and puberty with temperature information, and lifespan, ultimate total length and weight. These data may not be always available in the literature for many species. It also requires a consistent choice of the data and a careful establishment of assumptions (Saraiva et al. 2011), which is subject to physiological and biological knowledge of the studied organisms. Therefore, this method is suitable for species with sufficient information on both zero-variate data and pseudo-data, in which it would estimate the optimal parameters for a DEB model to best predict observations. However, it may potentially compromise the accuracy of parameters for species without these comprehensive datasets.

Temperature is particularly imperative in the physiological processes of the sea cucumber due to the unique characteristic of hibernation and aestivation. Because it is difficult to estimate parameters related to the Arrhenius relationship using the covariation method, these parameters were estimated following a standard procedure by van der Meer (2006). Although the sea cucumber can survive in a wide range of temperatures between -1.5 and $30.5^{\circ} \mathrm{C}$ in a short period (Zhang et al. 2004, Yang et al. 2005), the optimal range for growth is $10-15^{\circ} \mathrm{C}$ (Yu \& Song 1999). Temperatures beyond the optimal range would cause dramatic declines in physiological activities. Therefore, it is essential to obtain accurate information for parameters governing both lower and upper boundaries. Unfortunately, the published data have shown great variability (Liu et al. 1996, Li et al. 2002, Yang et al. 2005). A physiological measurement in the laboratory has recorded that the maximum feeding rate occurs at $20^{\circ} \mathrm{C}$, but was still reasonably high at $25^{\circ} \mathrm{C}$ (Yang et al. 2005). However, a field experiment has indicated that the feeding rate of $2 \mathrm{yr}$ old individuals decreased considerably from $19^{\circ} \mathrm{C}$, and all individuals stopped feeding when the temperature reached $22^{\circ} \mathrm{C}$ (Liu et al. 1996). Yu \& Song (1999) reported that the suitable temperature for growth is within $5-17^{\circ} \mathrm{C}$. 
This discrepancy may be related to experimental conditions and/or physiological differences between the juvenile and adult. Adults are less tolerant and therefore the triggering temperature for aestivation is lower than that for juveniles (Yu \& Song 1999, Yang et al. 2005). Despite evidence of size-dependent tolerance temperature, available information is not sufficient to extend the DEB model by incorporating a functional response. Furthermore, the feeding rate seems a quicker process than that of respiration at the upper boundary in the sea cucumber. A small increase in temperature may result in individuals completely stopping feeding, but continuing respiration (e.g. Liu et al. 1996). The rate of decrease in oxygen consumption at the upper boundary is considerably lower than that of the feeding rate ( $\mathrm{Li}$ et al. 2002). The difference in $T_{\mathrm{H}}$ values for feeding and respiration has been reported in Crassostrea gigas (Bourlès et al. 2009). Although there is some evidence in the rate of changes between feeding and maintenance in the sea cucumber, the available information is insufficient to be incorporated into the model in the present study. Further experiments are required to investigate whether this discrepancy is caused by the existence of different Arrhenius relationships or experimental artefacts.

When applying the model, it is noted that the development of eggs is not part of a standard DEB model and needs to be tested separately. As the extension of a standard DEB model was beyond the scope of this study, we did not include the topic of egg development. And the parameters for egg development may not be correctly estimated with the covariation method either. Additionally, existing datasets are insufficient to take energy content of eggs into consideration when doing the parameterisation, and so further experiments are required to gather information for such purpose.

Despite economic and ecological benefits of sea cucumber-shellfish co-culture (Yang et al. 2000, Yuan et al. 2015), optimal ratios of the sea cucumber and shellfish have been investigated through trial and error and experimentation (e.g. Zhou et al. 2006, Yu et al. 2014). This traditional method is not only costly but also inaccurate. For the present simulation dataset, the ratio of sea cucumbers to scallops and oysters was 1:35 and 1:12, respectively (Zhou et al. 2006). The size of scallops ( $3.0 \mathrm{~cm}$ shell length) was smaller than oysters $(7.7 \mathrm{~cm}$ shell length). Although it was not possible to calculate food availability for sea cucumbers from both cultures, the model simulation has shown that the scaled functional responses were $f=0.62$ and 0.51, respectively, for co-culture with scallops and oysters. This suggests that the experimental design has resulted in more biodeposits in co-culture with scallops than with oysters. Based on the growth rate, Zhou et al. (2006) concluded that the maximum density in each lantern net is 34 scallops. However, our modelling result indicated that 34 individuals would still result in approximately two-thirds of the maximum food density for sea cucumber growth. Similarly, the growth of the sea cucumber co-cultured with the scallop Chlamys farreri at a density of 1 individual $\mathrm{m}^{-2}$ was reported to be $60 \%$ faster than those at a density of 2 individuals $\mathrm{m}^{-2}$ (Yang et al. 2001), but the optimal ratio could not be determined with the experimental result. Because the optimal ratio depends on dynamics of environmental conditions and energetics of co-cultured species, full assessment and design of co-culture practices can only be determined using an IMTA model (Ren et al. 2012). The development of the sea cucumber DEB model has provided a tool for growth predictions of the sea cucumber in various environmental conditions and a basis for optimising stocking density of IMTA operations.

Acknowledgements. The study was supported by the New Zealand Foundation of Research, Science and Technology (Contract Number C01X0513), The National Science \& Technology Pillar Program of China (2011BAD13B06) and the National Natural Science Foundation of China (41076111; 41276172).

\section{LITERATURE CITED}

Alunno-Bruscia M, Bourlès Y, Maurer D, Robert S and others (2011) A single bio-energetics growth and reproduction model for the oyster Crassostrea gigas in six Atlantic ecosystems. J Sea Res 66:340-348

Barrington K, Chopin T, Robinson S (2009) Integrated multitrophic aquaculture (IMTA) in marine temperate waters. In: Soto D (ed) Integrated mariculture: a global review. FAO Fish Aquacult Tech Pap 529. FAO, Rome, p 7-46

* Bourlès Y, Alunno-Bruscia M, Pouvreau S, Tollu G and others (2009) Modelling growth and reproduction of the Pacific oyster Crassostrea gigas: advances in the oysterDEB model through application to a coastal pond. J Sea Res 62:62-71

Chen J (1990) Sea cucumber culture in China. In: Bueno P, Lovatelli A (eds) Brief introduction to mariculture of five selected species in China. FAO Corporate Document Repository, Rome

Chen J (2003) Overview of sea cucumber farming and sea ranching practices in China. SPC Beche-de-mer Inf Bull $18: 18-23$

Chopin T, Robinson SMC, Troell M, Neori A, Fang J (2008) Multitrophic integration for sustainable marine aquaculture. In: Jørgensen SE, Fath BD (eds) The encyclopedia of ecology, ecological engineering, Vol 3. Elsevier, Oxford, p 2463-2475

Hamano T, Amio M, Hayashi K (1989) Population dynamics 
of Stichopus japonicas Selenka (Holothuroidea, Echinodermata) in an intertidal zone and on the adjacent subtidal bottom with artificial reefs for Sargassum. Suisanzoshoku 37:179-186

Ito S, Kitamura H (1998) Technical development in seed production of the Japanese sea cucumber, Stichopus japonicus. SPC Beche-de-mer Inf Bull 10:24-28

Kooijman SALM (2010) Dynamic energy budget theory for metabolic organisation. Cambridge University Press, Cambridge

Lauerman LML, Smoak JM, Shaw TJ, Moore WS, Smith KL (1997) ${ }^{234} \mathrm{Th}$ and ${ }^{210} \mathrm{~Pb}$ evidence for rapid ingestion of settling particles by mobile epibenthic megafauna in the abyssal NE Pacific. Limnol Oceanogr 42:589-595

Li SK, Wang LC, Gao YG (2001) Co-culture of sea cucumbers and abalones in intertidal ponds. Fisheries Modernisation 2:16-18

Li B, Yang H, Zhang T, Zhou Y, Zhang C (2002) Effect of temperature on respiration and excretion of sea cucumber Apostichopus japonicus. Oceanol Limnol Sin 33: 182-187

Liao YL (1997) Fauna Sinica, Phylum Echinodermata, Class Holothuroidea. Science Press, Beijing

Lika K, Kearney MR, Freitas V, van der Veer HW and others (2011) The 'covariation method' for estimating the parameters of the standard dynamic energy budget model I: philosophy and approach. J Sea Res 66:270-277

Liu Y, Li F, Song B, Sun H, Zhang X, Gu B (1996) Study on aestivating habit of sea cucumber Apostichopus japonicas Selenka: ecological characteristics of aestivation. J Fish Sci China 3:41-48

* Miller RJ, Smith CR, DeMaster DJ, Fornes WL (2000) Feeding selectivity and rapid particle processing by deep-sea megafaunal deposit feeders: a ${ }^{234} \mathrm{Th}$ tracer approach. J Mar Res 58:653-673

Qiu T, Zhang T, Hamel JF, Mercier A (2015) Development, settlement and post-settlement growth. In: Yang $\mathrm{H}$, Hamel JF, Mercier A (eds) The sea cucumber Apostichopus japonicus: history, biology and aquaculture. Academic Press, London, p 111-130

Ren JS, Schiel DR (2008) A dynamic energy budget model: parameterisation and application to the Pacific oyster Crassostrea gigas in New Zealand waters. J Exp Mar Biol Ecol 361:42-48

Ren JS, Stenton-Dozey J, Plew DR, Fang J, Gall M (2012) An ecosystem model for optimising production in integrated multitrophic aquaculture systems. Ecol Modell 246:34-46

Rosland R, Strand $\varnothing$, Alunno-Bruscia M, Bacher C, Strohmeier T (2009) Applying dynamic energy budget (DEB) theory to simulate growth and bio-energetics of blue mussels under low seston conditions. J Sea Res 62:49-61

Saraiva S, van der Meer J, Kooijman SALM, Sousa T (2011) DEB parameters estimation for Mytilus edulis. J Sea Res 66:289-296

Saraiva S, van der Meer J, Kooijman SALM, Witbaard R, Philippart CJH, Hippler D, Parker R (2012) Validation of a dynamic energy budget (DEB) model for the blue mussel Mytilus edulis. Mar Ecol Prog Ser 463:141-158

Sibuet M, Segonzac M (1985) Abondance et répartition de l'épifaune mégabenthique. In: Laubier L, Monniot C (eds) Peuplements profonds du Golfe de Gascogne (Campagnes BIOGAS). IFREMER, Brest, p 143-156

Editorial responsibility: Gianluca Sará,

Palermo, Italy
Slater MJ, Jeffs AG, Carton AG (2009) The use of the waste from green-lipped mussels as a food source for juvenile sea cucumber Australostichopus mollis. Aquaculture 292:219-224

Sun G (2013) Shandong local standards - sea cucumber Apostichopus japonicus. www.docin.com/p-798281216. html (in Chinese)

van der Meer J (2006) An introduction to dynamic energy budget (DEB) models with special emphasis on parameter estimation. J Sea Res 56:85-102

van der Veer HW, Cardoso JFMF, van der Meer J (2006) The estimation of DEB parameters for various North Atlantic bivalve species. J Sea Res 56:107-124

* Yamana Y, Hamano T, Goshima S (2010) Natural growth of juveniles of the sea cucumber Apostichopus japonicus: studying juveniles in the intertidal habitat in Hirao Bay, eastern Yamaguchi Prefecture, Japan. Fish Sci 76: 585-593

Yang H, Wang J, Zhou Y, Zhang T, Wang P, He W (2000) Comparison of efficiencies of different culture systems in the shallow sea along Yantai. J Fish Sci China 24: 140-145

Yang $\mathrm{H}$, Zhou Y, Wang J, Zhang T, Wang P, He Y, Zhang F (2001) A modelling estimation of carrying capacities for Chlamys farreri, Laminaria japonica and Apostichopus japonicus in Sishiliwan Bay, Yantai, China. J Fish Sci China 7:27-31

Yang H, Yuan X, Zhou Y, Mao Y, Zhang T, Liu Y (2005) Effects of body size and water temperature on food consumption and growth in the sea cucumber Apostichopus japonicus (Selenka) with special reference to aestivation. Aquacult Res 36:1085-1092

Yu D, Song B (1999) Variation of survival rates and growth characteristics of pond cultural juvenile Apostichopus japonicus. J Fish Sci China 6:109-110

K Yu Z, Zhou Y, Yang H, Hu C (2014) Bottom culture of the sea cucumber Apostichopus japonicas Selenka (Echinodermata: Holothuroidea) in a fish farm, Southern China. Aquacult Res 45:1434-1441

Yuan X, Zhou Y, Mao Y (2015) Apostichopus japonicas: a key species in integrated polyculture systems. In: Yang H, Hamel JF, Mercier A (eds) The sea cucumber Apostichopus japonicus: history, biology and aquaculture. Academic Press, London, p 323-332

Zamora LN, Dollimore J, Jeffs AG (2014) Feasibility of coculture of the Australasian sea cucumber (Australostichopus mollis) with the Pacific oyster (Crassostrea gigas) in northern New Zealand. NZ J Mar Freshw Res 48: 394-404

Zhang T, Zhou W, Song Z (2004) Key technique in cultivation of sea cucumber (Apostichopus japonicus) using artificial reef with high yield and high benefit. Hebei Fisheries 3:71-75

Zhang L, Song X, Hamel JF, Mercier A (2015) Aquaculture, stock enhancement and restocking. In: Yang H, Hamel JF, Mercier A (eds) The sea cucumber Apostichopus japonicus: history, biology and aquaculture. Academic Press, London, p 289-322

Zhou Y, Yang H, Liu S, Yuan X and others (2006) Feeding and growth on bivalve biodeposits by the deposit feeder Stichopus japonicas Selenka (Echinodermata: Holothuroidea) co-cultured in lantern nets. Aquaculture 256:510-520

Submitted: May 17, 2016; Accepted: November 17, 2016

Proofs received from author(s): January 6, 2017 